\author{
Measurements of Time-Variable Gravity Show Mass Loss in \\ Antarctica \\ Isabella Velicogna and John Wahr \\ Science 311, 1754 (2006); \\ DOI: $10.1126 /$ science. 1123785
}

This copy is for your personal, non-commercial use only.

If you wish to distribute this article to others, you can order high-quality copies for your colleagues, clients, or customers by clicking here.

Permission to republish or repurpose articles or portions of articles can be obtained by following the guidelines here.

The following resources related to this article are available online at www.sciencemag.org (this information is current as of July 18, 2012 ):

Updated information and services, including high-resolution figures, can be found in the online version of this article at:

http://www.sciencemag.org/content/311/5768/1754.full.html

A list of selected additional articles on the Science Web sites related to this article can be found at:

http://www.sciencemag.org/content/311/5768/1754.full.html\#related

This article has been cited by 41 article(s) on the ISI Web of Science

This article has been cited by 13 articles hosted by HighWire Press; see:

http://www.sciencemag.org/content/311/5768/1754.full.html\#related-urls

This article appears in the following subject collections:

Atmospheric Science

http://www.sciencemag.org/cgi/collection/atmos 


\section{Measurements of Time-Variable Gravity Show Mass Loss in Antarctica}

Isabella Velicogna ${ }^{1,2 *}$ and John Wahr ${ }^{1 *}$

Using measurements of time-variable gravity from the Gravity Recovery and Climate Experiment satellites, we determined mass variations of the Antarctic ice sheet during 2002-2005. We found that the mass of the ice sheet decreased significantly, at a rate of $152 \pm 80$ cubic kilometers of ice per year, which is equivalent to $0.4 \pm 0.2$ millimeters of global sea-level rise per year. Most of this mass loss came from the West Antarctic Ice Sheet.

$\mathrm{T}$ he Antarctic ice sheet is Earth's largest reservoir of fresh water. Accurate estimates of its mass variability, accompanied by realistic error bars, would greatly reduce current uncertainties in projected sea-level change, with obvious societal and economic impacts. There have been substantial improvements in monitoring the ice sheet in the past few years $(1-3)$, although recent studies have provided contrasting mass balance estimates $(1,3)$.

Antarctic mass variability is difficult to measure because of the ice sheet's size and complexity. Previous estimates have used a variety of techniques (1), each with intrinsic limitations and uncertainties. A problem common to all these techniques is the difficulty of monitoring the entire ice sheet. Studies that rely on a single method can provide estimates for only a portion of the ice sheet, and even studies that synthesize results from several techniques suffer from sparse data in critical regions.

The most recent Intergovernmental Panel on Climate Change (IPCC) assessment estimated that the Antarctic contribution to sea-level rise during the past century was $0.2 \pm 0.3 \mathrm{~mm} /$ year (2). The report predicted that the Antarctic ice sheet will probably gain mass during the 21 st century because of increased precipitation in a warming global climate. Recent radar altimeter measurements (3) have shown an increase in the overall thickness of the East Antarctic Ice Sheet's (EAIS's) interior during 1992-2003. However, the IPCC prediction does not consider possible dynamic changes in coastal regions, and radar altimetry provides only sparse coverage of those areas (2). Detailed interferometric synthetic-aperture radar and airborne laser altimeter surveys of glaciers along the edge of the West Antarctic Ice Sheet (WAIS) show rapid increases in near-coastal discharge during the past few years (4). The overall contribution of the Antarctic ice sheet to global sea-level change thus depends on the balance between

${ }^{1}$ University of Colorado, Cooperative Institute for Research in Environmental Sciences and Department of Physics, University Campus Box 390, Boulder, CO 80309-0390, USA. ${ }^{2}$ ]et Propulsion Laboratory, California Institute of Technology, Mail Stop 300-233, Pasadena, CA 911098099, USA.

*To whom correspondence should be addressed. E-mail: isabella@colorado.edu (I.V.); wahr@colorado.edu (J.W.) mass changes in the interior and those in coastal areas (1). The gravitational survey of Antarctica provided by the Gravity Recovery and Climate Experiment (GRACE) satellites and discussed in this paper is a comprehensive survey of the entire ice sheet and is thus able to overcome the issue of limited sampling.

GRACE (5) provides monthly estimates of Earth's global gravity field at scales of a few hundred kilometers and larger. Time variations in the gravity field can be used to determine changes in Earth's mass distribution. GRACE mass solutions have no vertical resolution, however, and do not reveal whether a gravity variation over Antarctica is caused by a change in snow and ice on the surface, a change in atmospheric mass above Antarctica, or postglacial rebound (PGR: the viscoelastic response of the solid Earth to glacial unloading over the past several thousand years). Users must employ independent means to separate those contributions.

We used GRACE gravity-field solutions for 34 months between April 2002 and August 2005

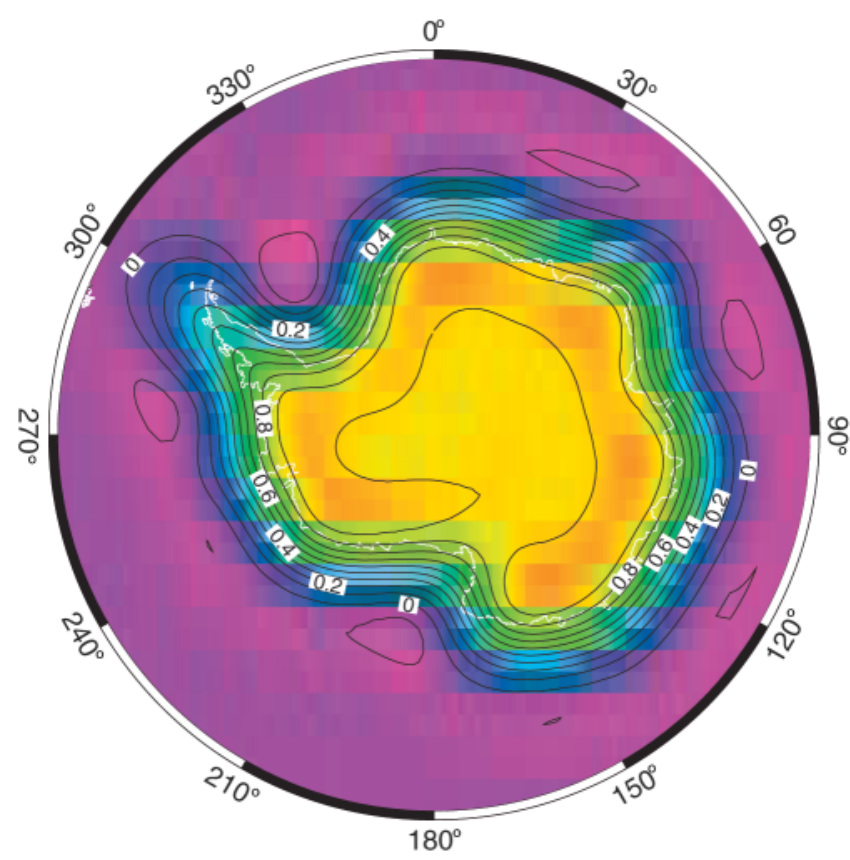

Fig. 1. The averaging function used to estimate the change in total Antarctic mass. 
Before interpreting the scaled results as ice sheet change, we had to address the issues of errors in the GRACE gravity fields and the contamination from other geophysical sources of gravity-field variability. To estimate the effects of errors, we convolved our averaging functions with uncertainty estimates for the GRACE Stokes coefficients (13). We obtained $1 \sigma$ error estimates that can be interpreted as $68.3 \%$ confidence intervals.

There are two types of geophysical contamination: one caused by signals outside Antarctica and the other from Antarctic signals unrelated to snow and ice. Leakage from outside Antarctica occurs because the averaging function extends beyond the boundaries of Antarctica. The leakage is increased because our omission of $l=1$ terms causes the averaging function to have a small-amplitude tail that extends around the globe.

We considered two sources of external leakage: continental hydrology outside Antarctica and ocean mass variability. The hydrologi- cal contamination was estimated using monthly global water storage fields from the Global Land Data Assimilation System (14). The ocean contamination was estimated using a Jet Propulsion Laboratory version of the Estimating the Circulation and Climate of the Ocean general circulation model (15). In both cases, we added a uniform layer to the global ocean so that the total land plus ocean mass was conserved at every time step. We removed the predicted hydrology leakage from the GRACE monthly mass estimates to obtain the monthly Antarctic mass estimates shown in Fig. 2. The predicted oceanic leakage was negligible and so was not removed.

The Antarctic mass change from GRACE shows a trend superimposed on shorter period variability (Fig. 2). We simultaneously fit a trend and annually and semiannually varying terms to the GRACE-minus-leakage results. Interpreting the trend as being due entirely to a change in ice, we inferred an ice volume increase of $39 \pm 14 \mathrm{~km}^{3} /$ year (the trend obtained

Fig. 2. GRACE monthly mass solutions for the Antarctic ice sheet for April 2002 to August 2005. Blue circles show results after removing the hydrology leakage. Red crosses show results after also removing the PGR signal. The latter represent our best estimates of the mass variability. The error bars include only the contributions from uncertainties in the GRACE gravity fields and represent $68.3 \%$ confidence intervals (13). Also shown is the linear trend that best fits the red crosses.

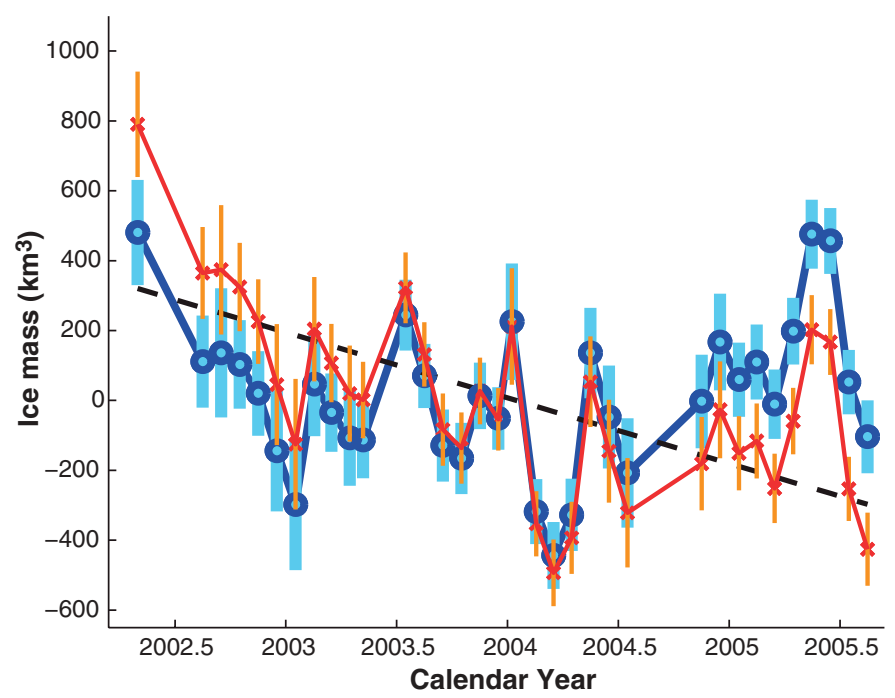

Fig. 3. Monthly ice mass changes and their bestfitting linear trends for WAIS (red) and EAIS (green) for April 2002 to August 2005. The GRACE data have been corrected for hydrology leakage and for PGR.

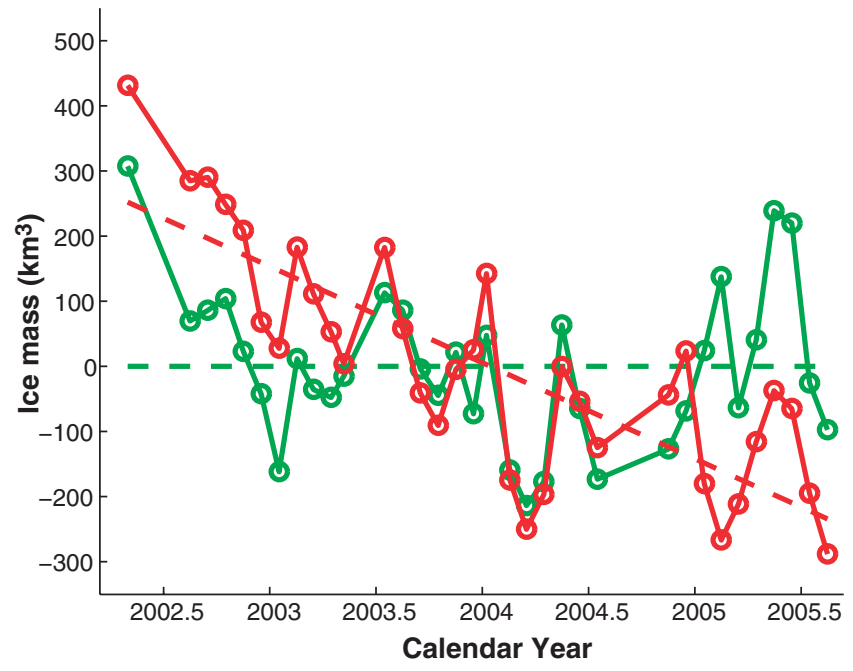

without removing the hydrology leakage is $51 \pm$ $14 \mathrm{~km}^{3} /$ year). The uncertainty reflects the errors in the GRACE gravity-field solutions and was computed using the GRACE monthly error bars (Fig. 2).

This ice mass estimate is contaminated by variations in atmospheric mass and from PGR. European Centre for Medium-Range Weather Forecasts (ECMWF) meteorological fields were used to remove atmospheric effects from the raw data before constructing gravity fields. But there are errors in those fields. We estimated the secular component of those errors by finding monthly differences between meteorological fields from ECMWF and from the National Centers for Environmental Prediction, applying the Antarctic averaging function to those differences and fitting a trend, and annually and semiannually varying terms to the results. The linear trend was small, equivalent to about 10 $\mathrm{km}^{3} /$ year, and was interpreted as the uncertainty due to atmospheric errors. We took the root sum square (RSS) with the effects of GRACE gravity-field errors, to obtain a new error estimate of $\pm 16 \mathrm{~km}^{3} /$ year.

A PGR signal is indistinguishable from a linear trend in ice mass. PGR effects are large and must be independently modeled and removed. There are two important sources of error in PGR estimates: the ice history and Earth's viscosity profile. We estimated the PGR contribution and its uncertainties using two ice history models: ICE-5G (16) and IJ05 (17). IJ05 is available only for Antarctica, so we combined it with ICE-5G outside Antarctica. We convolved these ice histories with viscoelastic Green's functions for an incompressible Earth (18). We computed trends in the Stokes coefficients for all plausible combinations of twolayer viscosity profiles and convolved these trends with the averaging function. ICE-5G trends are consistently larger than the IJ05 trends. We estimated the range of possible PGR contributions by defining our lower bound to be the minimum IJ05 trend (over all viscosity profiles) and our upper bound to be the maximum ICE-5G trend. Our best estimate of PGR trend is the midpoint of this range. This estimate translates to an apparent ice increase of $192 \pm 79 \mathrm{~km}^{3} /$ year, where the uncertainty corresponds to the bounds of our PGR range.

We subtracted this PGR contribution from the GRACE-minus-leakage ice mass estimates (Fig. 2). The best-fitting linear trend, and our final estimate of the decrease in total Antarctic mass between the summers of 2002 and 2005, is $152 \pm 80 \mathrm{~km}^{3} /$ year. The uncertainty is the RSS of the errors in the GRACE fit and in the PGR contribution. This rate of ice loss corresponds to $0.4 \pm 0.2 \mathrm{~mm} /$ year of global sealevel rise.

The PGR contribution (192 $\pm 79 \mathrm{~km}^{3} /$ year $)$ is much larger than the uncorrected GRACE trend (39 $\pm 14 \mathrm{~km}^{3} /$ year). A significant ice mass trend does not appear until the PGR contribu- 
tion is removed. The implication is that when averaged over all Antarctica, the gravity signals from PGR and from ice variability are closely coincident (with opposing signs), and it underscores the importance of obtaining a meaningful PGR uncertainty. Our uncertainty accommodates all plausible PGR contributions, and removing even the smallest such contribution still implies a loss of ice mass.

We also determined results for WAIS and EAIS separately (Fig. 3). We estimated the errors, the leakage, and the PGR contamination of each signal, as described above for the entire ice sheet. Both these ice sheets appear to have lost mass at higher rates during 2002-2004 than during 2004-2005; this is even more evident in the total Antarctic results (Fig. 2).

By fitting a trend and annual and semiannual terms to the WAIS and EAIS results, we find that most of the Antarctic mass loss comes from WAIS. After correcting for the hydrology leakage and the PGR signal, we obtain a WAIS mass loss of $148 \pm 21 \mathrm{~km}^{3} /$ year. The EAIS mass loss is $0 \pm 56 \mathrm{~km}^{3} /$ year. Because of its relatively large uncertainty, we are not able to determine whether EAIS is in balance or not. The final error bars for WAIS and EAIS, like those for all Antarctica, are dominated by the PGR uncertainty. The predicted PGR gravity signals at individual points in WAIS are actually somewhat larger than the PGR signals at EAIS points. The overall EAIS mass error is larger than that for WAIS simply because EAIS covers an area almost three times larger, so the EAIS averaging function is sensitive to the PGR signal integrated over a much larger area.

For these individual ice sheets, but unlike for all Antarctica, the PGR and ice mass signals do not cancel one another. For EAIS, the un- corrected GRACE trend is about equal to the PGR signal, and so we find no significant trend after removing PGR. For WAIS, the uncorrected GRACE trend and the PGR signal have about the same magnitudes but opposite signs, so the WAIS trend becomes even larger after PGR is removed.

The GRACE result for total Antarctic ice mass change includes complete contributions from such regions as the East Antarctic coastline and the circular cap south of $82^{\circ} \mathrm{S}$, which have not been completely surveyed with other techniques. The comprehensive nature of this result arises because a gravity signal at the altitude of GRACE is sensitive to mass variations averaged over a broad region of the underlying surface, not just at the point directly beneath the satellite. The main disadvantage of GRACE is that it is more sensitive than other techniques to PGR; in fact, our error estimates are dominated by PGR uncertainties. As more GRACE data become available, it will become feasible to search for long-term changes in the rate of mass loss. A change in the rate would not be contaminated by PGR errors, because the PGR rates will remain constant over the satellite's lifetime.

\section{References and Notes}

1. E. Rignot, R. Thomas, Science 297, 1502 (2002).

2. J. A. Church et al., in Climate Change 2001: The Scientific Basis, Third Assessment Report of the IPCC (Cambridge Univ. Press, Cambridge, 2001), pp. 639-694.

3. C. Davis, Y. Li, J. McConnell, M. Frey, E. Hanna, Science 308, 5730 (2005).

4. R. Thomas et al., Science 306, 255 (2004)

5. GRACE, launched in March 2002 and administered by NASA and the Deutsches Zentrum für Luft-und Raumfahrt, is mapping Earth's gravity field every 30 days during its 10- to 11-year lifetime. GRACE consists of two identical satellites in identical orbits, separated by $\sim 220 \mathrm{~km}$. The satellites use microwaves to monitor their separation distance. Onboard accelerometers and Global Positioning System receivers detect nongravitational accelerations and geocentric orbital motion.

6. M. Cheng, B. Tapley, J. Geophys. Res. 109, B09402 (2004)

7. J. Wahr, S. Swenson, V. Zlotnicki, I. Velicogna, Geophys. Res. Lett. 31, L11501 (2004)

8. B. Tapley, S. Bettadpur, M. Watkins, C. Reigber, Geophys. Res. Lett. 31, L09607 (2004)

9. I. Velicogna, J. Wahr, Geophys. Res. Lett. 32, L18505 (2005)

10. M. Tamisiea, E. Leuliette, ]. Davis, ]. Mitrovica, Geophys. Res. Lett. 32, L20501 (2005)

11. S. Swenson, ]. Wahr, P. C. D. Milly, Water Resour. Res. 39, 1223 (2003)

12. To determine the scaling factor for the entire ice sheet, we applied our averaging function to the gravitational signature of a uniform 1-cm water mass change spread evenly over the ice sheet. We obtained an estimate of $0.62 \mathrm{~cm}$. We thus multiplied each GRACE estimate by 1/0.62. Determining scaling factors for WAIS and EAIS is more complicated because the EAIS averaging function extends slightly over WAIS and vice versa. We applied each averaging function to a uniform mass change over each region individually and used the four resulting values to determine the linear combination of WAIS and EAIS results that correctly recovers the mass in each region.

13. J. Wahr, S. Swenson, I. Velicogna, Geophys. Res. Lett., in press.

14. M. Rodell et al., Bull. Am. Meteorol. Soc. 85, 381 (2004).

15. T. Lee, I. Fukumori, D. Menemenlis, Z. Xing, L. Fu, J. Phys. Oceanogr. 32, 1404 (2002)

16. W. R. Peltier, Annu. Rev. Earth Planet. Sci. 32, 111 (2004)

17. E. Ivins, T. S. James, Antarct. Sci. 17, 541 (2005)

18. I. Velicogna, ]. Wahr, J. Geophys. Res. 107, 2376 (2002)

19. We thank S. Bettadpur, ]. Cheng, J. Reis, E. Rignot, and M. Watkins for data and advice. This work was supported by NASA's Cryospheric and Solid Earth Programs and by the NSF Office of Polar Programs. This research was partially carried out at the Jet Propulsion Laboratory.

13 December 2005; accepted 21 February 2006

Published online 2 March 2006;

$10.1126 /$ science. 1123785

Include this information when citing this paper.

\section{Seasonality and Increasing Frequency of Greenland Glacial Earthquakes}

\author{
Göran Ekström, ${ }^{1 *}$ Meredith Nettles, ${ }^{2}$ Victor C. Tsai ${ }^{1}$
}

Some glaciers and ice streams periodically lurch forward with sufficient force to generate emissions of elastic waves that are recorded on seismometers worldwide. Such glacial earthquakes on Greenland show a strong seasonality as well as a doubling of their rate of occurrence over the past 5 years. These temporal patterns suggest a link to the hydrological cycle and are indicative of a dynamic glacial response to changing climate conditions.

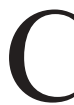
ontinuous monitoring of seismic waves recorded at globally distributed stations (1) has led to the detection and identification of a new class of earthquakes associated

${ }^{1}$ Department of Earth and Planetary Sciences, Harvard University, 20 Oxford Street, Cambridge, MA 02138, USA. ${ }^{2}$ Lamont-Doherty Earth Observatory of Columbia University, Palisades, NY 10964, USA.

*To whom correspondence should be addressed. E-mail: ekstrom@seismology.harvard.edu with glaciers $(2,3)$. These "glacial earthquakes" are characterized by emissions of globally observable low-frequency waves that are incompatible with standard earthquake models for tectonic stress release but can be successfully modeled as large and sudden glacial-sliding motions (4). Seismic waves are generated in the solid earth by the forces exerted by the sliding ice mass as it accelerates down slope and subsequently decelerates. The observed duration of sliding is typically 30 to $60 \mathrm{~s}$. All detected events of this type are associated with mountain glaciers in Alaska or with glaciers and ice streams along the edges of the Antarctic and Greenland ice sheets. The Greenland events are most numerous, and we present new data indicating a strong seasonality and an increasing frequency of occurrence for these events since at least 2002.

For the period January 1993 to October 2005, we have found 182 earthquakes on Greenland by analysis of continuous records from globally distributed seismic stations (5). None of these earthquakes are reported in standard seismicity catalogs. We have modeled seismograms for 136 of the best-recorded events to confirm their glacial-sliding source mechanism and obtain improved locations (Fig. 1) (6,7). This analysis yields an estimate of the twice-time-integrated active force couple at the earthquake source, a quantity that can be interpreted as the product of sliding mass and sliding distance $(2,8)$. All events have long-period seismic magnitudes in the range 4.6 to 5.1 , corresponding to a product 